06

\title{
Концепция оптимизации твердотельного аккумулятора водорода на основе магния для мобильного транспорта
}

\author{
(С) Б.М. Зыков, Т.М. Красненкова, Б.А. Лазба, А.И. Марколия \\ Сухумский физико-технический институт АН Абхазии, \\ Сухум, Абхазия \\ I e-mail: kras1946@mail.ru
}

Поступило в Редакцию 11 декабря 2019 г.

В окончательной редакции 11 декабря 2019 г.

Принято к публикации 11 января 2019 г.

Изучены порошки магния с малыми добавками других металлов для выяснения оптимальных условий гидрирования и дегидрирования с целью применения в качестве твердотельного аккумулятора водорода для мобильного транспорта. Найден оптимальный состав с весовой формулой $\mathrm{Mg}_{10} \mathrm{Ni}$. Только этот состав при циклировании не подвержен деградации обратимой водородной емкости. Установлено, что максимальной обратимой емкости соответствует минимальная температура гидрирования. Предложена концепция выбора оптимальной добавки к магнию, обоснованная на атомном и электронном уровнях.

Ключевые слова: водород, аккумулятор, магний, добавка, состав смеси, гидрирование, оптимизация, концепция.

DOI: 10.21883/JTF.2020.06.49288.395-19

\section{Введение}

Уменьшение на Земле разведанных запасов нефти, значительное удорожание ее поиска и добычи на шельфе, а также все возрастающее ухудшение экологии от увеличения выбросов в атмосферу отработанных газов двигателями внутреннего сгорания (ДВС), в особенности в городах с населением более 1 млн. чел., которых становится все больше, отсутствие в ближайшем будущем альтернативы автомобильному транспорту приводят к необходимости поиска для ДВС другого экологически чистого топлива. Им, вне конкуренции, является водород $\left(\mathrm{H}_{2}\right)$ [1-8]. Его запасы, в частности, в составе воды на Земле практически неисчерпаемы $[9,10]$. Что касается экологичности, то продуктом сгорания $\mathrm{H}_{2}$ в воздухе и кислороде является вода.

Но применение $\mathrm{H}_{2}$ в баллонах высокого давления или в жидком виде, во-первых, экономически не выгодно, так как в твердотельных гидридных системах при тех же массогабаритных характеристиках для обеспечения одного и того же пробега (обычно $300-400 \mathrm{~km}$ ) без дозаправки можно запасти больше $\mathrm{H}_{2}$ [11]. Во-вторых, применение баллонов высокого давления $(P)$ на 70-100 MРа для сохранения необходимого для этого пробега запаса $\mathrm{H}_{2}$ представляет большую опасность при дорожно-транспортных происшествиях (ДТП), а применение жидкого $\mathrm{H}_{2}$ требует дорогого и сложного криогенного оборудования, обеспечивающего безопасное хранение $\mathrm{H}_{2}$ в отсутствие его расхода из-за самопроизвольного повышения температуры $(T)$ и, как следствие, $P_{\mathrm{H}_{2}}$ из-за орто-пара перехода [11]. Следовательно, для мобильного транспорта (маневровые тепловозы, автодрезины, автомобильный, шахтный, внутрискладской и, в перспективе, возможно, авиационный [4] транспорт) более всего подходят твердотельные аккумуляторы $\mathrm{H}_{2}[4,8,11-19]$.

\section{1. Требования к аккумуляторам водорода}

Исходя из того, что удельная теплота сгорания $\mathrm{H}_{2}$ (в cal $/ \mathrm{kg}$ ) в три раза выше, чем у бензина и дизельного (в том числе и биодизельного) топлива, и что 70-90\% энергии топлива в ДВС теряется, применение в автомобилестроении энергосберегающих водородных технологий может стать хорошим способом дальнейшего совершенствования автомобиля. В настоящее время наиболее привлекательно применение $\mathrm{H}_{2}$ в качестве как основного, так и дополнительного топлива для ДВС. Например, даже $10 \%$ добавка $\mathrm{H}_{2}$ к бензину позволяет поднять топливную экономичность на $30-40 \%$ и, самое главное, снизить уровень токсичности выхлопных газов, улучшив экологическую обстановку в крупных городах [20].

Выбор твердотельной системы для хранения $\mathrm{H}_{2}$ должен обеспечивать минимальную массу при удовлетворительных эксплуатационных характеристиках. При этом большую роль при создании аккумуляторов $\mathrm{H}_{2}$ для мобильного транспорта играют следующие параметры:

- удельный вес аккумулирующего $\mathrm{H}_{2}$ вещества;

- обратимая водородная емкость вещества (процент выхода $\mathrm{H}_{2}$ );

- температура гидрирования $\left(T_{\mathrm{hyd}}\right)$;

- давление $\mathrm{H}_{2}$ при гидрировании $\left(P_{\mathrm{H}_{2}}\right)$;

- время гидрирования; 
- температура выделения основной части (80-90\%) $\mathrm{H}_{2}$. Это одна из важнейших характеристик аккумулятора;

- температура выхлопных газов. В зависимости от числа оборотов двигателя может изменяться от 375 до $725 \mathrm{~K}$;

— равновесное давление дегидрирования (не менее $0.1 \mathrm{MPa})$;

- время (скорость) выделения $\mathrm{H}_{2}$;

- температура активации аккумулирующего состава (не выше $725 \mathrm{~K}$ );

- число циклов гидрирование-дегидрирование без снижения характеристик (ресурс аккумулятора);

- безопасность в аварийных ситуациях.

\section{2. Поиск аккумулирующего состава}

На начальном этапе исследований особое внимание уделялось низкотемпературным составам, поглощающим $\mathrm{H}_{2}$ в интервале $295-365 \mathrm{~K}$. Наиболее приемлемыми оказались интерметаллические соединения весовых составов типов $\mathrm{AB}_{5}(\mathrm{~A}-\mathrm{La}$, мишметалл из смеси редкоземельных металлов $\mathrm{Mm}, \mathrm{Ce}$; В - Fe, Co, Ni, $\mathrm{Cu}, \mathrm{Mn}, \mathrm{Al}) ; \mathrm{AB}_{2}(\mathrm{~A}-\mathrm{Ti}, \mathrm{Zr} ; \mathrm{B}-\mathrm{Mn}, \mathrm{V}, \mathrm{Cr}$, $\mathrm{Fe})$; $\mathrm{AB}(\mathrm{A}-\mathrm{Ti}, \mathrm{Zr} ; \mathrm{B}$ - Fe, Co) и композиты на основе V [11,14-25]. Например, в Германии и США были созданы экспериментальные автомобили на составах FeTi и $\mathrm{LaNi}_{5}$ [15]. Но из-за того, что обратимая водородная емкость всех упомянутых составов в оптимуме не превышала 3 mass \%, вес так называемого гидридного бака был сравним с весом самого автомобиля. Кроме того, из-за высокой стоимости лучшего из них $\mathrm{LaNi}_{5}$ (\$17 за kg в ценах 1980 г.) стало ясно, что с такими параметрами невозможно массовое производство подобных автомобилей. Поэтому в некоторых странах в качестве условия для открытия государственного финансирования соответствующих проектов были установлены критерии по массовому содержанию $\mathrm{H}_{2}$ в материале. Так, например, в Японии - это 5.5\%, в США - 6\%, в России - 6.5\% [22]. Следует отметить, что последний выбор не был обоснован экспериментально. В России наилучшее содержание $\mathrm{H}_{2}$ в $5.5 \%$ было получено для смеси $\operatorname{Mg}(72 \%) \operatorname{Mm}(8 \%) \mathrm{Ni}(20 \%) \quad[21,24] \quad$ и $5.8 \%$ для сплава $\mathrm{Mg}_{10} \mathrm{Ni}(80.6 \% \mathrm{Mg}+19.4 \% \mathrm{Ni})$ [26]. Поэтому ясно, что следует переходить к высокотемпературным аккумулирующим составам.

\section{3. Магний как основная компонента аккумулирующего состава}

Первым трем вышеперечисленным требованиям лучше всего отвечает магний $(\mathrm{Mg})$. Вначале считалось, что основную роль в накоплении $\mathrm{H}_{2}$ играет гидрид магния $\mathrm{MgH}_{2}$. Это широко известное в неорганической химии соединение $[12,16,19,27-32]$. Но оказалось, что компактный $\mathrm{Mg}$ в объем $\mathrm{H}_{2}$ не поглощает, и его надо измельчать в порошок. Но из-за сильной окислительной способности это надо делать в инертных средах аргоне (Ar), водороде или органических растворителях.

Было установлено, что гидрид $\mathrm{MgH}_{2}$ содержит 7.6 mass. $\% \mathrm{H}_{2}$. Он диссоциирует начиная с 555-565 K на $\mathrm{Mg}$ и $\mathrm{H}_{2}$. Растворимость $\mathrm{H}_{2}$ в $\mathrm{Mg}$ возрастает с $T$ и достигает атомной доли 0.1 при $835 \mathrm{~K}$. Но $\mathrm{Mg}$ при этой $T$ уже ощутимо испаряется. $P$ паров $\mathrm{Mg}$ при этой $T$ уже составляет $\sim 1.33 \cdot 10^{2} \mathrm{~Pa}$. Температура же плавления $\operatorname{Mg}\left(T_{\text {fus }}\right)$ составляет $923 \mathrm{~K}$ [33]. Начиная с $T=565 \mathrm{~K} \mathrm{Mg}$ может быть прогидрирован полностью при $P_{\mathrm{H}_{2}}=0.1 \mathrm{MPa}$ за $10 \mathrm{~h}$. Но он перед этим должен быть превращен в порошок с оптимальным размером частиц в 30-50 $\mu \mathrm{m}$. Кроме того, порошок $\mathrm{Mg}$ должен быть активирован в вакууме при $675-775 \mathrm{~K}$ в течение $1-2$ h. Цель этой операции - удаление или, по крайней мере, растрескивание оксидной пленки и удаление адсорбированной воды (так называемое обезгаживание). При соблюдении этих условий затем гарантируется получение водородной емкости в $80-90 \%$ от рассчитанной. Эту емкость принято измерять в литрах на килограмм $(1 / \mathrm{kg})$ вещества или в массовых \%. Процесс активирования порошка можно значительно ускорить применяя циклический отжиг в вакууме в присутствии $\mathrm{H}_{2}$ при $P_{\mathrm{H}_{2}} \sim 0.05-0.1 \mathrm{MPa}$. Цикличность необходима для того, чтобы в одном цикле гарантированно удалить хотя бы один монослой воды, образующейся при восстановлении $\mathrm{Mg}$ из его оксида $\mathrm{MgO}$. При этом производится отжиг порошка в $\mathrm{H}_{2}$ при $700-725 \mathrm{~K}$ в течение трех минут c последующей откачкой $\mathrm{H}_{2}$ без снижения $T$. Для очистки $\mathrm{Mg}$ от оксидов или углеродных загрязнений (в последнем случае образуется метан $\mathrm{CH}_{4}$, который и откачивается) обычно достаточно 30-50 циклов.

Однако не следует считать, что $\mathrm{H}_{2}$ запасается в $\mathrm{Mg}$ только в виде $\mathrm{MgH}_{2} . \mathrm{H}_{2}$ проникает в металл $(\mathrm{Me})$, отдавая один электрон с каждого атома в зону проводимости Ме, занятую его делокализованными $s$-электронами, и диффундируя в него уже в виде протона. Поэтому при наличии сплошной пленки $\mathrm{MgH}_{2}$ (также, как и $\mathrm{MgO}$ ) диффузия $\mathrm{H}_{2}$ в $\mathrm{Mg}$ прекращается, так как при наличии насыщенных химических связей в пленках вновь поступающему $\mathrm{H}_{2}$ уже некуда отдавать свои электроны. Следовательно, 7.6 mass.\% $\mathrm{H}_{2}$ в $\mathrm{Mg}$ после образования сплошной пленки $\mathrm{MgH}_{2}$ никогда не были бы получены. Однако на практике получается и боо́льшая водородная емкость. Например, в этой работе нами при $T_{\text {hyd }}=685 \mathrm{~K}$ за $7 \mathrm{~h}$ при $P_{\mathrm{H}_{2}}=2.5 \mathrm{MPa}$ и $T$ дегидрирования $635-700 \mathrm{~K}$ получена обратимая емкость в 7.8 mass.\% $\mathrm{H}_{2}$ (8691/kg). Для уменьшения времени гидрирования $P_{\mathrm{H}_{2}}$ увеличивали до $5 \mathrm{MPa}$ (табл. 1-3). $P_{\mathrm{H}_{2}}$ изменяли таким образом, чтобы экспозиция в $\mathrm{H}_{2}$ (т.е. произведение $P \cdot t$, где $t$ - время гидрирования) во всех случаях была приблизительно одинаковой. Следовательно, поскольку весь $\mathrm{Mg}$ (а порошок - это тоже твердое тело) не может превратиться в гидрид, основная часть поглощенного $\mathrm{H}_{2}$ определяется его адсорбцией на стенках дефектов, абсорбцией в объемных дефектах и окклюзией. Последнее 
Таблица 1. Максимальный выход $\mathrm{H}_{2}$ из сплавов на основе $\mathrm{Mg}$

\begin{tabular}{|c|c|c|c|c|}
\hline $\begin{array}{c}\text { Состав (mass.), } \\
d=0.9 \mathrm{~g} / \mathrm{cm}^{3}\end{array}$ & $\begin{array}{c}\text { Мах выход, } \\
\text { 1/kg }\end{array}$ & $\begin{array}{c}\text { Мax выход, } \\
\text { mass. \% }\end{array}$ & $\begin{array}{c}\text { Выход } \\
\text { отн. } \mathrm{Mg}, \%\end{array}$ & $\begin{array}{l}\text { Условия гидрирования: } \\
T, \mathrm{~K} ; t, \mathrm{~h} ; P, \mathrm{MPa}\end{array}$ \\
\hline $\mathrm{Mg}$ & 869 & 7.81 & 100 & $685 \mathrm{~K}, 7 \mathrm{~h}, 2.5 \mathrm{MPa}$ \\
\hline $\mathrm{Mg}_{2} \mathrm{Ni}$ & 443 & 3.98 & 51.0 & $660 \mathrm{~K}, 7 \mathrm{~h},-^{\prime \prime}-$ \\
\hline $\mathrm{Mg}_{4} \mathrm{Ni}$ & 565 & 5.08 & 65.0 & $660 \mathrm{~K}, 7 \mathrm{~h},-^{\prime \prime}-$ \\
\hline $\mathrm{Mg}_{6} \mathrm{Ni}$ & 449 & 4.03 & 51.7 & $660 \mathrm{~K}, 7 \mathrm{~h},-^{\prime \prime}-$ \\
\hline $\mathrm{Mg}_{10} \mathrm{Ni}$ & 643 & 5.78 & 74.0 & $660 \mathrm{~K}, 7 \mathrm{~h},-^{\prime \prime}-$ \\
\hline $\mathrm{Mg}_{14} \mathrm{Ni}$ & 638 & 5.74 & 73.4 & $660 \mathrm{~K}, 7 \mathrm{~h},-^{\prime \prime}-$ \\
\hline $\mathrm{Mg}_{20} \mathrm{Ni}$ & 636 & 5.71 & 73.2 & $660 \mathrm{~K}, 7 \mathrm{~h},-^{\prime \prime}-$ \\
\hline $\mathrm{Mg}_{3} \mathrm{Fe}$ & 319 & 2.87 & 36.8 & $675 \mathrm{~K}, 6 \mathrm{~h}, 3 \mathrm{MPa}$ \\
\hline $\mathrm{Mg}_{5} \mathrm{Fe}$ & 314 & 2.83 & 36.3 & $675 \mathrm{~K}, 6 \mathrm{~h},-^{\prime \prime}-$ \\
\hline $\mathrm{Mg}_{10} \mathrm{Fe}$ & 487 & 4.37 & 56.0 & $660 \mathrm{~K}, 7 \mathrm{~h}, 2.5 \mathrm{MPa}$ \\
\hline $\mathrm{Mg}_{2} \mathrm{Cu}$ & 291 & 2.61 & 33.5 & $660 \mathrm{~K}, 7 \mathrm{~h},-^{\prime \prime}-$ \\
\hline $\mathrm{Mg}_{6} \mathrm{Cu}$ & 592 & 5.32 & 68.2 & $660 \mathrm{~K}, 7 \mathrm{~h},-^{\prime \prime}-$ \\
\hline $\mathrm{Mg}_{10} \mathrm{Cu}$ & 627 & 5.63 & 72.2 & $660 \mathrm{~K}, 7 \mathrm{~h}-{ }^{\prime \prime}-$ \\
\hline $\mathrm{Mg}_{14} \mathrm{Cu}$ & 598 & 5.37 & 68.8 & $660 \mathrm{~K}, 7 \mathrm{~h},-^{\prime \prime}-$ \\
\hline $\mathrm{Mg}_{3} \mathrm{Al}$ & 462 & 4.15 & 53.2 & $685 \mathrm{~K}, 5 \mathrm{~h}, 3.5 \mathrm{MPa}$ \\
\hline $\mathrm{Mg}_{5} \mathrm{Al}$ & 576 & 5.18 & 66.3 & $685 \mathrm{~K}, 5 \mathrm{~h},-^{\prime \prime}-$ \\
\hline $\mathrm{Mg}_{10} \mathrm{Al}$ & 588 & 5.29 & 67.7 & $685 \mathrm{~K}, 5 \mathrm{~h},-^{\prime \prime}-$ \\
\hline $\mathrm{Mg}_{10} \mathrm{Ni}_{0.5} \mathrm{Cu}_{0.5}$ & 608 & 5.46 & 70.0 & $660 \mathrm{~K}, 7 \mathrm{~h}, 2.5 \mathrm{MPa}$ \\
\hline $\mathrm{Mg}_{10} \mathrm{Ni}_{0.5} \mathrm{Fe}_{0.5}$ & 605 & 5.44 & 69.7 & $660 \mathrm{~K}, 7 \mathrm{~h},-^{\prime \prime}-$ \\
\hline $\mathrm{Mg}_{10} \mathrm{Ni}_{0.5} \mathrm{Ti}_{0.5}$ & 546 & 4.90 & 62.8 & $660 \mathrm{~K}, 7 \mathrm{~h},-^{\prime \prime}-$ \\
\hline $\mathrm{Mg}_{10} \mathrm{Fe}_{0.5} \mathrm{Ti}_{0.5}$ & 542 & 4.87 & 62.4 & $685 \mathrm{~K}, 7 \mathrm{~h},-^{\prime \prime}-$ \\
\hline $\mathrm{Mg}_{10} \mathrm{AlCu}$ & 448 & 4.03 & 51.6 & $685 \mathrm{~K}, 6 \mathrm{~h}, 3 \mathrm{MPa}$ \\
\hline $\mathrm{Mg}_{10} \mathrm{AlFe}$ & 430 & 3.86 & 49.5 & $685 \mathrm{~K}, 5 \mathrm{~h}, 3.5 \mathrm{MPa}$ \\
\hline $\mathrm{Mg}_{10} \mathrm{AlNi}$ & 378 & 3.39 & 43.5 & $685 \mathrm{~K}, 5 \mathrm{~h},-^{\prime \prime}-$ \\
\hline $\mathrm{Mg}_{10} \mathrm{AlTi}$ & 358 & 3.21 & 41.2 & $685 \mathrm{~K}, 6 \mathrm{~h}, 3 \mathrm{MPa}$ \\
\hline $\mathrm{Mg}_{10} \mathrm{AlZn}$ & 354 & 3.18 & 40.7 & $660 \mathrm{~K}, 6 \mathrm{~h},-^{\prime \prime}-$ \\
\hline $\mathrm{Mg}_{10} \mathrm{AlNb}$ & 349 & 3.13 & 40.1 & $660 \mathrm{~K}, 6 \mathrm{~h},-^{\prime \prime}-$ \\
\hline
\end{tabular}

явление представляет значительное накопление $\mathrm{H}_{2}$ в закрытых порах материала [19] (естественно, перекрытых чистым Ме) после его адсорбции на стенках пор, приводящее к значительному увеличению $P_{\mathrm{H}_{2}}$ внутри пор (до сотен и тысяч МРа). Это вызывает растрескивание материала - так называемую водородную коррозию, или „водородную болезнь“ металлов. Этим, в частности, и объясняется значительное увеличение дисперсности порошка (уменьшение размера его зерен) и „распухание“ брикета, достигающее после обезгаживания, активирования в $\mathrm{H}_{2}$ и 7 циклов гидрированиедегидрирование 100\% первоначального объема.

Хотя с уменьшением размера зерен порошка растет его поверхность и водородная емкость вроде бы должна увеличиваться, тем не менее от цикла к циклу (гидрирование-дегидрирование) при всех прочих равных внешних условиях она в основном деградирует. Это можно объяснить ухудшением теплопроводности порошка из-за увеличения его дисперсности и, как следствие, выходом его из оптимального режима гидрирования. Отсюда следует, что не нужно стремиться к чрезмерной дисперсности порошка уже на стадии его приготовления. Таким образом, окклюзия играет существенную роль в поглощении $\mathrm{H}_{2}$ материалом, и термин „гидрирование“ при этом становится в большой степени условным.
T.е. под ним теперь следует понимать все процессы поглощения $\mathrm{H}_{2}$, а не только образование химического соединения - гидрида.

Для существования явления окклюзии закрытая пора, как указано выше, с внешней стороны должна быть перекрыта чистым Ме или токопроводящей пленкой, иначе становятся невозможными диссоциация молекул $\mathrm{H}_{2}$, его последующая автоионизация и поглощение в объем материала. Поэтому операция восстановления $\mathrm{Mg}$ перед гидрированием становится необходимой.

\section{4. Выбор состава для водородного аккумулятора}

Исходя из $T_{\max }$ выхлопных газов ДВС, оптимальная $T_{\text {hyd }}$ чистого $\mathrm{Mg}(685 \mathrm{~K})$, естественно, является очень высокой. Поэтому с помощью добавок к нему других веществ (в основном Ме) пытаются снизить оптимальную $T_{\text {hyd }}$ c желательным сохранением большой обратимой водородной емкости. Если снижения температуры почти всегда удается достичь, то практика показывает, что любая добавка к $\mathrm{Mg}$ уменьшает водородную емкость состава таким образом, как будто часть его исключается из гидрирования. Причина этого рассмотрена ниже. 
Таблица 2. Максимальный выход $\mathrm{H}_{2}$ из смесей на основе $\mathrm{Mg}$

\begin{tabular}{|c|c|c|c|c|}
\hline $\begin{array}{l}\text { Состав (mass.), } \\
d=0.9 \mathrm{~g} / \mathrm{cm}^{3}\end{array}$ & $\begin{array}{c}\text { Мах выход, } \\
1 / \mathrm{kg}\end{array}$ & $\begin{array}{l}\text { Мах выход, } \\
\text { mass. \% }\end{array}$ & $\begin{array}{c}\text { Выход } \\
\text { отн. } \mathrm{Mg}, \%\end{array}$ & $\begin{array}{l}\text { Условия гидрирования } \\
T, \mathrm{~K} ; t, \mathrm{~h}, ;, \mathrm{MPa}\end{array}$ \\
\hline $\mathrm{Mg}$ & 869 & 7.81 & 100 & $685 \mathrm{~K}, 7 \mathrm{~h}, 2.5 \mathrm{MPa}$ \\
\hline $\mathrm{Mg}_{3} \mathrm{Fe}$ & 459 & 4.12 & 52.8 & $675 \mathrm{~K}, 6.5 \mathrm{~h}, 2.75 \mathrm{MPa}$ \\
\hline $\mathrm{Mg}_{5} \mathrm{Fe}$ & 586 & 5.27 & 67.5 & $685 \mathrm{~K}, 7 \mathrm{~h}, 2.5 \mathrm{MPa}$ \\
\hline $\mathrm{Mg}_{7} \mathrm{Fe}$ & 683 & 6.14 & 78.6 & $685 \mathrm{~K}, 7 \mathrm{~h},-^{\prime \prime}-$ \\
\hline $\mathrm{Mg}_{9} \mathrm{Fe}$ & 709 & 6.37 & 81.6 & $685 \mathrm{~K}, 7 \mathrm{~h},-^{\prime \prime}-$ \\
\hline $\mathrm{Mg}_{10} \mathrm{Fe}$ (Fe не восст.) & 559 & 5.02 & 64.4 & $685 \mathrm{~K}, 7 \mathrm{~h},-^{\prime \prime}-$ \\
\hline $\mathrm{Mg}_{10} \mathrm{Fe}\left(\mathrm{Fe}\right.$ восст. в $\left.\mathrm{H}_{2}\right)$ & 712 & 6.39 & 81.9 & $685 \mathrm{~K}, 7 \mathrm{~h},-^{\prime \prime}-$ \\
\hline $\mathrm{Mg}_{12} \mathrm{Fe}$ & 748 & 6.72 & 86.1 & $675 \mathrm{~K}, 6.5 \mathrm{~h}, 2.75 \mathrm{MPa}$ \\
\hline $\mathrm{Mg}_{14} \mathrm{Fe}$ & 638 & 5.73 & 73.4 & $675 \mathrm{~K}, 7.5 \mathrm{~h}, 2.5 \mathrm{MPa}$ \\
\hline $\mathrm{Mg}_{10} \mathrm{Ni}$ & 714 & 6.41 & 82.1 & $650 \mathrm{~K}, 7 \mathrm{~h},-^{\prime \prime}-$ \\
\hline $\mathrm{Mg}_{10} \mathrm{X} 18 \mathrm{H} 10 \mathrm{~T}$ & 703 & 6.32 & 80.9 & $675 \mathrm{~K}, 6.5 \mathrm{~h}, 2.75 \mathrm{MPa}$ \\
\hline $\mathrm{Mg}_{10} \mathrm{~V}$ & 601 & 5.40 & 69.2 & $680 \mathrm{~K}, 7 \mathrm{~h},-^{\prime \prime}-$ \\
\hline $\mathrm{Mg}_{10} \mathrm{Ti}$ & 542 & 4.87 & 62.4 & $660 \mathrm{~K}, 4.5 \mathrm{~h}, 4 \mathrm{MPa}$ \\
\hline $\mathrm{Mg}_{20} \mathrm{Pd}$ & 695 & 6.24 & 80.0 & $660 \mathrm{~K}, 8 \mathrm{~h}, 2.5 \mathrm{MPa}$ \\
\hline $\mathrm{Mg}_{8} \mathrm{Fe}(\mathrm{CO})_{5}$ & 398 & 3.58 & 45.9 & $660 \mathrm{~K}, 6 \mathrm{~h}, 3 \mathrm{MPa}$ \\
\hline $\mathrm{Mg}_{10} \mathrm{Fe}(\mathrm{CO})_{5}$ & 480 & 4.32 & 55.4 & $650 \mathrm{~K}, 5 \mathrm{~h}, 3.5 \mathrm{MPa}$ \\
\hline $\mathrm{Mg}_{16} \mathrm{Fe}(\mathrm{CO})_{5}$ & 513 & 4.61 & 59.1 & $660 \mathrm{~K}, 5 \mathrm{~h},-^{\prime \prime}-$ \\
\hline $\mathrm{Mg}_{24} \mathrm{Fe}(\mathrm{CO})_{5}$ & 517 & 4.65 & 59.6 & $660 \mathrm{~K}, 7 \mathrm{~h}, 2.5 \mathrm{MPa}$ \\
\hline $\mathrm{Mg}_{20} \mathrm{FeTi}$ & 735 & 6.61 & 84.6 & $685 \mathrm{~K}, 9 \mathrm{~h},-^{\prime \prime}-$ \\
\hline $\mathrm{Mg}_{20} \mathrm{FeV}$ & 541 & 4.86 & 62.3 & $660 \mathrm{~K}, 7.5 \mathrm{~h},-^{\prime \prime}-$ \\
\hline $\mathrm{Mg}_{20} \mathrm{FeNi}$ & 671 & 6.03 & 77.2 & $685 \mathrm{~K}, 7 \mathrm{~h},-^{\prime \prime}-$ \\
\hline $\mathrm{Mg}_{20} \mathrm{NiTi}$ & 668 & 6.01 & 77.0 & $685 \mathrm{~K}, 3.5 \mathrm{~h}, 5 \mathrm{MPa}$ \\
\hline $\mathrm{Mg}_{20} \mathrm{NiCu}$ & 643 & 5.78 & 74.0 & $675 \mathrm{~K}, 7 \mathrm{~h}, 2.5 \mathrm{MPa}$ \\
\hline $\mathrm{Mg}_{20} \mathrm{NiV}$ & 434 & 3.90 & 50.0 & $650 \mathrm{~K}, 7 \mathrm{~h},-^{\prime \prime}-$ \\
\hline $\mathrm{Mg}_{20} \mathrm{NiX} 18 \mathrm{H} 10 \mathrm{~T}$ & 582 & 5.23 & 67.0 & $660 \mathrm{~K}, 7 \mathrm{~h},-^{\prime \prime}-$ \\
\hline $\mathrm{Mg}_{20} \mathrm{TiV}$ & 631 & 5.67 & 72.6 & $660 \mathrm{~K}, 8 \mathrm{~h},-^{\prime \prime}-$ \\
\hline $\mathrm{Mg}_{20} \mathrm{VX} 18 \mathrm{H} 10 \mathrm{~T}$ & 476 & 4.28 & 54.8 & $660 \mathrm{~K}, 8 \mathrm{~h},-^{\prime \prime}-$ \\
\hline $\mathrm{Mg}_{30} \mathrm{NiPd}$ & 568 & 5.10 & 65.0 & $660 \mathrm{~K}, 7.5 \mathrm{~h},-^{\prime \prime}-$ \\
\hline
\end{tabular}

Таблица 3. Зависимость максимального выхода $\mathrm{H}_{2}$ от начальной плотности брикета для смеси $\mathrm{Mg}_{10} \mathrm{Ni}$

\begin{tabular}{|c|c|c|c|c|c|c|}
\hline \multicolumn{3}{|c|}{ Начальные условия } & \multirow{2}{*}{$\begin{array}{l}\text { Мах } \\
\text { выход, } \\
1 / \mathrm{kg}\end{array}$} & \multirow{2}{*}{$\begin{array}{l}\text { Мах выход, } \\
\text { mass. \% }\end{array}$} & \multirow{2}{*}{$\begin{array}{c}\text { Выход } \\
\text { отн. Mg, } \\
\%\end{array}$} & \multirow{2}{*}{$\begin{array}{c}\text { Условия } \\
\text { гидрирования: } \\
T, \mathrm{~K} ; t, \mathrm{~h}, ; P, \mathrm{MPa}\end{array}$} \\
\hline $\begin{array}{c}\text { Избыточное } P, \\
\text { МРа }\end{array}$ & $\begin{array}{l}T, \\
\mathrm{~K}\end{array}$ & $\begin{array}{c}D, \\
\mathrm{~g} / \mathrm{cm}^{3}\end{array}$ & & & & \\
\hline $\mathrm{Mg}-1.0$ & 295 & 0.902 & 869 & 7.81 & 100 & $685 \mathrm{~K}, 7 \mathrm{~h}, 2.5 \mathrm{MPa}$ \\
\hline 0.0 & 295 & 0.482 & 663 & 5.96 & 76.3 & $650 \mathrm{~K}, 4.5 \mathrm{~h}, 4 \mathrm{MPa}$ \\
\hline 1.0 & 295 & 0.902 & 672 & 6.04 & 77.4 & $650 \mathrm{~K}, 4 \mathrm{~h}, 4.5 \mathrm{MPa}$ \\
\hline 2.5 & 295 & 1.453 & 663 & 5.95 & 76.3 & $635 \mathrm{~K}, 6 \mathrm{~h}, 3 \mathrm{MPa}$ \\
\hline 3.5 & 295 & 1.604 & 668 & 6.00 & 76.9 & $635 \mathrm{~K}, 3 \mathrm{~h}, 5.5 \mathrm{MPa}$ \\
\hline 4.5 & 295 & 1.701 & 646 & 5.80 & 74.3 & $630 \mathrm{~K}, 6 \mathrm{~h}, 3 \mathrm{MPa}$ \\
\hline 5.0 & 295 & 1.722 & 646 & 5.80 & 74.3 & $635 \mathrm{~K}, 6 \mathrm{~h},-^{\prime \prime}-$ \\
\hline 6.0 & 295 & 1.732 & 646 & 5.80 & 74.3 & $635 \mathrm{~K}, 6 \mathrm{~h},-^{\prime \prime}-$ \\
\hline 6.5 & 295 & 1.758 & 687 & 6.17 & 79.1 & $650 \mathrm{~K}, 5.5 \mathrm{~h}, 3.25 \mathrm{MPa}$ \\
\hline 5.5 & 525 & 1.770 & 710 & 6.38 & 81.7 & $650 \mathrm{~K}, 6 \mathrm{~h}, 3 \mathrm{MPa}$ \\
\hline 6.0 & 525 & 1.790 & 699 & 6.28 & 80.4 & $650 \mathrm{~K}, 6 \mathrm{~h},-^{\prime \prime}-$ \\
\hline 2.5 & 705 & 1.835 & 681 & 6.12 & 78.4 & $650 \mathrm{~K}, 5 \mathrm{~h}, 3.5 \mathrm{MPa}$ \\
\hline 3.5 & 705 & 1.878 & 683 & 6.13 & 78.6 & $650 \mathrm{~K}, 6 \mathrm{~h}, 3 \mathrm{MPa}$ \\
\hline 4.5 & 705 & 2.008 & 666 & 5.98 & 76.7 & $650 \mathrm{~K}, 5.5 \mathrm{~h}, 3.25 \mathrm{MPa}$ \\
\hline 5.5 & 705 & 2.089 & & нет поглощең & & $650 \mathrm{~K}, 6 \mathrm{~h}, 3 \mathrm{MPa}$ \\
\hline
\end{tabular}


Исходя из этой компромиссной ситуации и руководствуясь к тому же экономическими соображениями и массогабаритными характеристиками, нами изучен следующий ряд добавок к $\mathrm{Mg}$ с различными отчасти обоснованными массовыми соотношениями: $\mathrm{Fe}, \mathrm{Ni}, \mathrm{Cu}, \mathrm{Zn}$, $\mathrm{Al}, \mathrm{Ti}, \mathrm{V}, \mathrm{Nb}, \mathrm{Pd}$, нержавеющая сталь марки X18H10T, карбонил железа $\mathrm{Fe}(\mathrm{CO})_{5}$ в виде порошков той же дисперсности, что и $\mathrm{Mg}$. Добавки In, $\mathrm{La}, \mathrm{Ce}, \mathrm{Eu}, \mathrm{Pt}$ не применялись из экономических соображений, хотя для сравнения применялась добавка $\mathrm{Pd}$ как наиболее растворяющего $\mathrm{H}_{2}$ материала. Применялись также двойные добавки из перечисленных Ме в различных их сочетаниях и соотношениях с Mg. Результаты по максимальной водородной емкости приведены в табл. 1 и 2.

\section{5. Методы исследования}

\section{1. Приготовление порошков и смесей}

Для приготовления аккумулирующих $\mathrm{H}_{2}$ составов применяется $\mathrm{Mg}$ чистотой $99.9 \%$, полученный помолом в вихревой мельнице стружки от фрезерования слитка с соблюдением правил вакуумной гигиены. При этом содержание в полученном порошке зерна методом ситового анализа составляет: $400 \mu \mathrm{m}-0.6 \%, 315 \mu \mathrm{m}-$ $80.7 \%, 200 \mu \mathrm{m}-10.1 \%, 160 \mu \mathrm{m}-3.5 \%, 63 \mu \mathrm{m}-$ $4.5 \%$, остальное - более $400 \mu \mathrm{m}$ и менее $50 \mu \mathrm{m}-$ $0.6 \%$. Добавка Ni представляет собой мелкодисперсный $(\leq 30 \mu \mathrm{m})$ порошок анодного электролитического $\mathrm{Ni}$ марки ПНЭ-1 чистотой 95\%. Ті и $\mathrm{Zr}$ порошки готовятся аналогично $\mathrm{Mg}$ из слитков, полученных в вакууме по газофазной йодидной технологии. Порошок $\mathrm{Cu}$ восстановлен в вакууме в среде $\mathrm{H}_{2}$ до появления красной окраски. Fe опилки фирмы Schering A.G., Berlin предварительно подвергаются помолу в той же мельнице, а затем восстанавливаются в вакууме в $\mathrm{H}_{2}$. Процесс восстановления - циклический при $T=715 \pm 10 \mathrm{~K}$ и $P_{\mathrm{H}_{2}}=(2.66-4.0) \cdot 10^{4} \mathrm{~Pa}$ в течение $3 \mathrm{~min}$ с последующей откачкой $\mathrm{H}_{2}$ до $P_{\text {res }} \sim 1.33 \mathrm{~Pa}$ без снижения $T$. Процедура восстановления производится в заранее откачанном до $P \leq 6.65 \cdot 10^{-1}$ Ра объеме. Число циклов составляет $\sim 50$. Порошок $\mathrm{V}$ получен помолом монокристаллической стружки в той же мельнице, а $\mathrm{Pd}-$ размолом обрезков тонкостенной трубки $\varnothing 2 \times 0.1 \mathrm{~mm}$ и $\mathrm{Zn}$ - из электродного материала. Порошок Al получен аналогично $\mathrm{Mg}$ из слитка чистотой $99.9 \%$. Применяется также мелкодисперсный $(\leq 30 \mu \mathrm{m})$ порошок карбонила железа $\mathrm{Fe}(\mathrm{CO})_{5}$ химической чистоты. От попытки применения $\mathrm{Zr}$ пришлось отказаться, так как из-за большого содержания в нем $\mathrm{O}$, скорее всего, из-за недостаточного вакуума при газофазном йодидном процессе его получения, при помоле в вихревой мельнице для создания состава с Mg эта смесь самовоспламеняется с детонацией.

\section{2. Приготовление сплавов}

Смесь помещается в контейнер из нержавеющей стали марки Х18H10T, утрамбовывается до достижения плотности $d=0.9 \mathrm{~g} / \mathrm{cm}^{3}$ начиная с насыпной $d=0.48 \mathrm{~g} / \mathrm{cm}^{3}$ и затем закрывается невакуумноплотной крышкой из стальной жести той же марки, что и контейнер. Последний помещается в вакуумный контейнер из такой же стали, который способен прогреваться в наружной муфельной печи. Перед плавкой шихты или напуском $\mathrm{H}_{2}$ для гидрирования смеси с $\mathrm{Mg}$ производится обезгаживание состава при $T=725 \mathrm{~K}$ до достижения вакуума лучше $1 \mathrm{~Pa}$, затем циклическая очистка в $\mathrm{H}_{2}$ при $P_{\mathrm{H}_{2}}=0.05-0.1 \mathrm{MPa}$ и $T=700-725 \mathrm{~K}$. При приготовлении сплавов плавка шихты производится при $T=1025-1075 \mathrm{~K}$ в течение $1.5 \mathrm{~h}$ под давлением аргона $P_{\mathrm{Ar}} \sim 2 \mathrm{MPa}$ в предварительно откачанном объеме. После самопроизвольного охлаждения контейнер со сплавом извлекается, сплав высверливается, его стружка подвергается помолу в указанной выше мельнице и затем готовится состав с плотностью $d=0.9 \mathrm{~g} / \mathrm{cm}^{3}$ в другом подобном контейнере с крышкой из указанной нержавеющей стали. Для исследований берется $5 g$ состава.

\section{3. Экспериментальный прибор}

На рисунке приведена схема экспериментального прибора. В вакуумном контейнере применено медное уплотнение типа Conflat, в вакуумных кранах - также медные типа конус-цилинр, в кранах для напуска газов плоские из фторопласта. Измерительный объем (3.891) и вакуумные коммуникации из нержавеющей стали $\mathrm{X} 18 \mathrm{H} 10 \mathrm{~T}$ обезгажены после изготовления и в процессе дальнейшей работы не обезгаживаются, так как больше контакта с атмосферой не имеют. Кроме измерительного, в работе учитываются также объемы вакуумных коммуникаций, кранов и манометров, открытые при

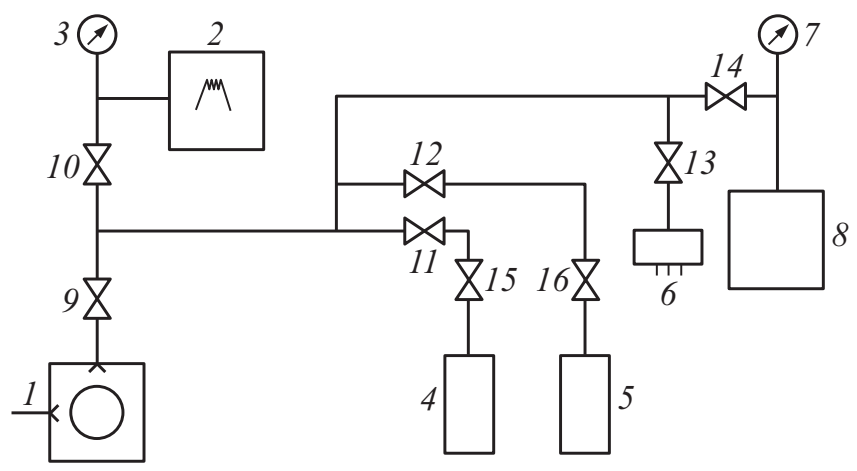

Схема установки для гидрирования порошков: $1-$ форвакуумный насос, 2 - муфельная печь, 3 - манометр высокого давления $(5 \mathrm{MPa}), 4$ - баллон с водородом, 5 - баллон с аргоном, 6 - датчик низкого давления $\left(1.33 \cdot 10^{-2} \mathrm{~Pa}\right), 7-$ манометр низкого давления $(0.1 \mathrm{MPa}), 8-$ измерительный объем (3.891), 9-14 - вакуумные клапаны, 15, 16 - редукторы. 
дегидрировании. В измерительном объеме применяется мановакуумметр типа 11201(250 делений на 0.1 MPa), а при измерениях в вакууме - датчик ПМТ-6-3М-1 с вакуумметром типа ВИТ-19ИТ-1. $T$ составов измеряется с помощью трех хромель-алюмелиевых термопар как усредненная из трех различных точек контейнера с составом с точностью $1 \mathrm{~K}$ приборами 24461A Кeysight (2 входа) и Fluke 179, а $T$ окружающей среды - прецизионным ртутным термометром с точностью $0.1 \mathrm{~K}$. При гидрировании применяется манометр МП4-У на $5 \mathrm{MPa}$ с точностью $2.5 \cdot 10^{-2} \mathrm{MPa}$. Для откачки применяется форвакуумный насос типа 2НВР5ДМ.

\section{4. Гидрирование}

После установления какой-либо из выбранных $T$ гидрирования - 575, 605, 635, 650, 660, 675 или $685 \mathrm{~K}$ в откачанный до $P \leq 1 \mathrm{~Pa}$ вакуумный контейнер напускается $\mathrm{H}_{2}$. В зависимости от поставленной задачи, $P_{\mathrm{H}_{2}}$ варьируется от 2.5 до $5 \mathrm{MPa}$, а время гидрирования от 3 до 9 часов. Применяется $\mathrm{H}_{2}$ марки В второго сорта чистотой не хуже 95\%. После экспозиции в $\mathrm{H}_{2}$ состав в контейнере самопроизвольно охлаждается, а $\mathrm{H}_{2}$ затем откачивается. Далее перекрывается откачка, открывается заранее откачанный измерительный объем и производится последовательный нагрев состава до прекращения изменения $P_{\mathrm{H}_{2}}$ в этом объеме при каждой из выбранных $T_{h}=475,525,555,575,585,675$ и $705 \mathrm{~K}$ с фиксацией величины $P_{\mathrm{H}_{2}}$ по измерительному мановакуумметру (в делениях) и окружающей $T_{r}\left(\right.$ в $\left.{ }^{\circ} \mathrm{C}\right)$. Для каждой из этих $T_{h}$ производится расчет водородной емкости в $1 /$ кg и в mass. \%, приведенной к нормальным условиям $(P=0.1 \mathrm{MPa}, T=273 \mathrm{~K})$ по формулам (1) и (2). Установлено, что повышение $T_{h}$ при дегидрировании до $725 \mathrm{~K}$ с большой точностью уже не приводит к увеличению выхода $\mathrm{H}_{2}$. Поэтому выход $\mathrm{H}_{2}$ при $705 \mathrm{~K}$ считается полным.

\section{5. Определение водородной емкости}

Водородная емкость (удельный объем) при дегидрировании определяется по формуле

$$
V_{\mathrm{H}_{2}}=\left(P_{\text {beg }}-P_{\text {fin }}\right) / P_{\text {beg }} \cdot V_{C} \cdot 273 /\left(273+T_{\mathrm{r}}\right) \cdot 1000 / P_{m},
$$

где $V_{\mathrm{H}_{2}}$ - удельный объем выделившегося $\mathrm{H}_{2}$, [1/kg]; $P_{\text {beg }}$ - начальное показание (остаточное давление) измерительного манометра типа 11201 , делений; $P_{\text {fin }}-$ конечное показание измерительного манометра, делений; $V_{C}-$ измерительный объем, [1]; $T_{r}$ - окружающая $T,\left[{ }^{\circ} \mathrm{C}\right] ; P_{m}-$ вес состава, $[\mathrm{g}]$.

Массовое содержание $\mathrm{H}_{2}$ в составе рассчитывается по формуле

$$
H_{m}=\rho \cdot V_{\mathrm{H}_{2}} / 1000 \cdot 100 \%,
$$

где $H_{m}-$ массовое содержание $\mathrm{H}_{2},[\%] ; \rho-$ плотность $\mathrm{H}_{2}$, [g/l] (0.08987); $V_{\mathrm{H}_{2}}$ - удельный объем выделившегося водорода, $[1 / \mathrm{kg}]$.

\section{6. Результаты исследования}

Во-первых, установлено, что механические смеси порошков с плотностью $0.9 \mathrm{~g} / \mathrm{cm}^{3}$ при насыпной плотности $0.48 \mathrm{~g} / \mathrm{cm}^{3}$ дают в большой степени сходные результаты по оптимальной $T_{\text {hyd }}$ и водородной емкости, даже смеси - несколько лучшие (табл. 1 и 2). Это позволяет значительно упростить процесс приготовления состава, исключив плавку шихты в вакууме под давлением Ar и последующее измельчение сплава.

Во-вторых, исследуя составы с различными весовыми формулами $\mathrm{Mg}_{n} \mathrm{X}$, где $n=2,3,4,5,6,7,9,10,12,14,20$ и 30 , а X - добавка, удалось установить, что оптимальной весовой формулой является $\mathrm{Mg}_{10} \mathrm{X}$, что упрощает задачу, исключая другие соотношения в смеси (табл. 1 и 2). Т.е. содержание добавки должно, в зависимости от ее плотности, составлять от 16 до 22\%. Наиболее исследованный в литературе состав $\mathrm{Mg}_{2} \mathrm{Ni}$ при этом показывает наихудшие результаты по водородной емкости. Следует отметить, что другие соотношения, кроме $\mathrm{Mg}_{2} \mathrm{X}$, в литературе почти неизвестны.

Руководствуясь требованиями, предъявляемыми к величинам водородной емкости в США (6 mass \%), укажем лишь те составы, для которых нами получена бо́льшая емкость. К ним относятся: $\mathrm{Mg}_{12} \mathrm{Fe}$ (6.72\%), $\mathrm{Mg}_{20} \mathrm{FeTi} \quad(6.61 \%), \quad \mathrm{Mg}_{10} \mathrm{Ni} \quad$ (6.41), $\quad \mathrm{Mg}_{10} \mathrm{X} 18 \mathrm{H} 10 \mathrm{~T}$ (6.32\%), $\mathrm{Mg}_{20} \mathrm{FeNi}(6.03 \%)$ и $\mathrm{Mg}_{20} \mathrm{NiTi}$ (6.01\%). При этом для всех составов, кроме $\mathrm{Mg}_{10} \mathrm{Ni}$, оптимальная $T_{\text {hyd }}$ составляет $675 \mathrm{~K}$ (для $\mathrm{Mg}_{20} \mathrm{FeTi}-685 \mathrm{~K}$ ) и лишь для $\mathrm{Mg}_{10} \mathrm{Ni}-648 \mathrm{~K}$. Температура же почти полного (90\%) выхода $\mathrm{H}_{2}$ для всех исследованных составов составляет $625 \mathrm{~K}$. Следует отметить, что максимальная емкость выхода $\mathrm{H}_{2}$ (обратимая) для всех составов, кроме $\mathrm{Mg}_{10} \mathrm{Ni}$, сохраняется только для первого цикла гидрирования, деградируя после 5 цикла более, чем на $10 \%$. И только для $\mathrm{Mg}_{10} \mathrm{Ni}$ после 23 циклов не отмечено деградации емкости. Поэтому последующие испытания этого состава на ресурс, занявшие 2 месяца работы, были прекращены.

Причины деградации емкости до конца не ясны до сих пор. Как оказалось на примере $\mathrm{Mg}_{10} \mathrm{Ni}$, они заключаются не только в потере составом теплопроводности из-за увеличения его дисперсности при гидрировании (водородной коррозии) и его „распухания“, достигающего через 7 циклов 100\% начального объема - ведь условия эксплуатации всех составов одинаковы. По-видимому, причина все же состоит в весовых соотношениях составов. Об этом достаточно убедительно свидетельствуют результаты исследования системы $\mathrm{Mg}_{n} \mathrm{Ni}$ (табл. 1). Чтобы разобраться в первой причине, для выяснения влияния начальной плотности состава на водородную емкость проведены специальные исследования с составом $\mathrm{Mg}_{10} \mathrm{Ni}$ (табл. 3), которые показали, что в широких пределах плотности практически не существует зависимости между нею и емкостью до тех пор, пока состав не начинает приобретать свойства массивного твердого тела $\left(d>2 \mathrm{~g} / \mathrm{cm}^{3}\right)$. В последнем случае из-за 
образования на поверхности в меру сплошной пленки химического соединения (не важно - оксида или гидрида) и уменьшения количества пор растворимость $\mathrm{H}_{2}$ в объеме прекращается. На то, что это было известно и ранее, указано выше.

Поскольку в настоящее время нам не известны методы исследования порошков, которые бы прояснили причины деградации их водородной емкости, нам представляется, что следует пойти статистическим путем, исследуя составы с гидридообразующими добавками; с добавками, не образующими гидридов; с сильными поглотителями $\mathrm{H}_{2}$, например, с Рd (табл. 2); с сильными поглотителями кислорода (например, $\mathrm{Ag}$ ), поскольку О является наиболее активной компонентой остаточных газов в вакууме, парциальное $P_{\mathrm{O}}$ которого лишь не более, чем на $1.5-2$ порядка величины меньше $P_{\text {res, }}$ с учетом $T_{\text {hyd }}$ и дегидрирования, являющихся оптимальными и для окисления Ме, и величины парциального $P_{\mathrm{O}}$ в рабочем вакууме, вполне достаточной для окисления Ме [34-36]; с сильными восстановителями (раскислителями, например, Mn); с наиболее инертными к кислороду элементами (например, Аu) и т. п. Таким образом, можно приблизиться и к пониманию того, в чем состоит влияние на состав химически почти подобных элементов, например, Fe, Co и $\mathrm{Ni}$. Только поняв причины процессов деградации можно пытаться ими управлять.

\section{7. Роль добавки и возможные принципы оптимизации составов на основе магния}

В литературе нам не известны подробные описания роли добавки к $\mathrm{Mg}$. Некоторые авторы считают ее раскислителем, другие - катализатором, причем без разъяснения процесса катализа на уровне переноса электронов. Достоверно установлены лишь два противоречивых факта ее влияния - снижение оптимальной $T_{\text {hyd }}$ и уменьшение водородной емкости по сравнению с чистым $\mathrm{Mg}$. Хотя последний факт говорит о том, что добавка как бы исключает часть $\mathrm{Mg}$ из процесса образования гидрида $\mathrm{MgH}_{2}$, вносящего, как и окклюзия, вклад в накопление $\mathrm{H}_{2}$.

Попробуем разобраться в роли добавки на атомном уровне. Установлено, что лучше всего работают добавки из переходных Ме, т.е. имеющих на уровне атомов вакантные и (или) полузаполненные (лучше первые) $d$-орбитали, т.е. Me III, IV, V, VI, VII и VIII b-групп Периодической системы элементов. С другой стороны, выше показано, что основную роль в накоплении $\mathrm{H}_{2}$ в твердом $\mathrm{Mg}$ (порошок - это тоже твердое тело) играет не образование гидрида $\mathrm{MgH}_{2}$, а окклюзия, т.е. накопление $\mathrm{H}_{2}$ в закрытых порах [19]. Но при этом со стороны поверхности по́ра должна быть перекрыта чистым Ме (в общем случае — проводящей пленкой). В противном случае, т.е. при наличии на поверхности пленки химического соединения с насыщенными связями (например, оксида или гидрида, что в данном случае наиболее вероятно), молекулярный $\mathrm{H}_{2}$, во-первых, не сможет на поверхности Ме матрицы диссоциировать на атомы (все химические взаимодействия чужеродных частиц с твердым телом начинаются именно с поверхности - особого состояния вещества [34-36]) и, во-вторых, атом Н затем не сможет отдать свой единственный $1 s$-электрон в зону проводимости Ме, образованную его делокализованными $s$-электронами, т.е. подвергнутся автоионизации. Только после этого оставшийся протон, согласно закону Кулона, может диффундировать в объем Ме, в том числе и в его кристаллическую решетку. Но для этого необходимо сохранение поверхностью жаростойкости, т.е. инертности к окислению, поскольку, как указано выше, через пленку оксида $\mathrm{H}_{2}$ не проходит. Это тем более важно, что $T_{\text {hyd }}$ и дегидрирования являются оптимальными и для окисления Ме [34-36].

Жаростойкость Ме сплавов (например, сталей) достигается следующим образом. В расплав (для равномерного распределения компонент) основного Ме вводится малая добавка (или несколько их для получения еще и необходимых механических свойств) какого-либо из переходных Ме, имеющих дополнительно к полузаполненным (по правилу Хунда) еще и вакантные $d$-орбитали (например, Sc, Y, La, Ti, Zr, Hf, $\mathrm{V}, \mathrm{Nb}, \mathrm{Ta})$ или из лантаноидов, имеющих вакантные $f$-орбитали (Ce, $\mathrm{Pr}, \mathrm{Nd}, \mathrm{Pm}, \mathrm{Sm})$ с целью образования одной или двух донорно-акцепторных связей с одной или двумя полностью заполненными $s p^{3}$-гибридными орбиталями атомов О. Две же другие полузаполненные $s p^{3}$-орбитали атома О образуют химические связи с полузаполненными орбиталями двух соседних атомов переходного Ме (в данном случае Fe). Известно, что при вступлении в химическую связь внешняя электронная конфигурация изолированного атома $\mathrm{O}-2 s^{2} 2 p^{4}$, для выравнивания энергии электронов в орбиталях с целью образования максимально возможного числа химических связей гибридизируется в 4 одинаковых по форме $s p^{3}$-гибридные орбитали - две заполненные и две полузаполненные. Эти $s p^{3}$-гибридные орбитали в результате кулоновского взаимного отталкивания приобретают в образующейся молекуле оксида уже тетраэдрическую внешнюю электронную конфигурацию. Две полузаполненные $s p^{3}$-орбитали О всегда могут образовать две ковалентные связи с двумя соседними атомами переходного Ме (трехцетровую мостиковую связь), отчего О и считается в основном двухвалентным. Но при наличии у других соседних атомов другого переходного Ме (или этого же) вакантных $d$-орбиталей появляется возможность дополнительного образования еще одной или двух донорно-акцепторных химических связей. И тогда О может стать уже 3- или 4-валентным. Таким образом, учитывая, что на поверхности переходного Ме из-за релаксации ее верхнего слоя атомов (экспериментально установлено, что на $10-12 \%)$, всегда у атома такого 
Ме есть еще и вакантная $d$-орбиталь, такой Ме, даже не имея для изолированного атома вакантных (хотя бы даже одной) орбиталей, может при взаимодействии с О образовывать (хотя бы на поверхности) субоксид типа $\mathrm{Me}_{3} \mathrm{O}$, а имеющий хотя бы одну вакантную орбиталь типа $\mathrm{Me}_{4} \mathrm{O}$ [37], причем в последнем случае даже в объемной кристаллической решетке [38]. Последним явлением и объясняется сильная растворимость О в $\mathrm{Sc}$, Y, La и лантаноидах (в последних за счет вакантных $f$-обиталей), Ti, $\mathrm{Zr}, \mathrm{Hf}, \mathrm{V}, \mathrm{Nb}$ и Та и отсутствие ее глубже второго слоя атомов в совершенных монокристаллах Cr, Мо и W [39]. Установлено, что совершенный монокристалл $\mathrm{W}$ с внешней электронной конфигурацией изолированных атомов $5 d^{4} 6 s^{2}$ не растворяет кислород, как $\mathrm{Cr}\left(3 d^{5} 4 s^{1}\right)$ и Мо $\left(4 d^{5} 5 s^{1}\right)$ (имеющие „провал“ одного $s$-электрона в $d$-оболочку), глубже второго слоя атомов подложки. Поэтому следует считать, что при образовании твердого тела из изолированных атомов, приводящем к уменьшению энергии, и у атомов W происходит „провал“ $s$-электрона, приводящий их к конфигурации $5 d^{5} 6 s^{1}$. Растворимость же О в кристаллической решетке Ме связана, как указано выше, с наличием у атомов последнего вакантных и достаточно локализованных орбиталей. На практике установлена релаксация верхнего слоя атомов на плотноупакованной грани (110) монокристалла $\mathrm{W}$ на $10-12 \%$. С другой стороны, также установлено наличие на этой грани вакантной $d$-орбитали, вероятнее всего, перпендикулярной грани, т. е. $d_{\mathrm{Z}} 2$, т.е. провал поверхностного $d$-электрона из полузаполненной орбитали в такую же объемную. T.е. первое связано с уменьшением энергии при образовании твердого тела вообще, а на любой поверхности в частности, а второе является следствием первого. Нам представляется, что не существует ограничений для этого эффекта и для других граней монокристалла, т.е. вообще для поверхности переходных Ме. Т.е. растворимость О под верхним слоем их атомов обеспечена всегда. Это подтверждается установленным явлением реконструкции поверхности переходных Ме при их взаимодействии с О вне зависимости от характера заполнения $d$-орбиталей [34-36,38,39]. При наличии же малой добавки другого переходного Ме таким же образом может образовываться интерметаллический субоксид, в зависимости от числа вакантных $d$-орбиталей добавки типов $\mathrm{Me}_{2}{ }^{\mathrm{I}} \mathrm{Me}^{\mathrm{II}} \mathrm{O}$ или $\mathrm{Me}_{2}{ }^{\mathrm{I}} \mathrm{Me}_{2}{ }^{\mathrm{II}} \mathrm{O}$ [40]. Из-за большой насыщенности химических связей $(\mathrm{O}-3$ - или 4-валентен), субоксиды металлов высокотермоустойчивы и диссоциируют часто только вблизи $T_{\text {fus }}$ основного Ме [34-37,40,41]. Из-за того, что часть атомов основного $\mathrm{Me}\left(16.7 \%\right.$ для типа $\mathrm{Me}_{2}{ }^{\mathrm{I}} \mathrm{Me}_{2}{ }^{\mathrm{II}} \mathrm{O}$ и $25 \%$ для $\left.\mathrm{Me}_{2}{ }^{\mathrm{I}} \mathrm{Me}{ }^{\mathrm{II}} \mathrm{O}[34,35,38]\right)$ теперь связана химически с металлом добавки, первый теряет способность к окислению, а распределенной и связанной добавки также мало для ее окисления, сплав в результате становится жаростойким, не теряя при этом электропроводности, т.к. $s$-электроны обоих Ме не вступают в химическую связь, и поэтому делокализованы. Таким образом, при нали- чии дефектов и в особенности закрытых пор материал обладает способностью к автоионизации чужеродных атомов, в частности, сохраняет способность пропускать в объем $\mathrm{H}_{2}$.

Образование субоксида на основе $\mathrm{Mg}$ происходит несколько иным способом, но по тем же принципам. Атом Mg с внешней электронной конфигурацией $3 s^{2}$, имея 3 вакантные $3 p$-орбитали с повышенной относительно $3 s$-оболочки энергией при образовании химического соединения переходит в возбужденное состояние, образуя 3 гибридные $s p$-орбитали, одна из которых вакантна, а две - полузаполнены. Последние легко образуют двойную связь с двумя полузаполненными $s p^{3}$-орбиталями атома О. При этом образуется молекула оксида $\mathrm{MgO}$ с одной вакантной $s p$-орбиталью $\mathrm{Mg}$ и двумя заполненными $s p^{3}$-орбиталями О. Легко видеть, что, добавляя к $\mathrm{MgO}$ переходной Ме с вакантными $d$-орбиталями и, в зависимости от их числа, образуя донорно-акцепторную связь с одной или двумя заполненными $s p^{3}$-орбиталями атома О, можно получить молекулу субоксида типов $\mathrm{MgOMe} \mathrm{или} \mathrm{MgOMe}_{2}$ с учетом того, что донорно-акцепторные связи обычно не бывают двойными [42]. За счет делокализации $s$-электронов добавки сохраняется электропроводность субоксида, что необходимо для поглощения $\mathrm{H}_{2}$.

Но у атомов добавки остаются еще неспаренные $d$-электроны (полузаполненные $d$-орбитали), способные к образованию химической связи, поэтому образовавшееся соединение нельзя считать высшим оксидом. Эта связь с О исключается из-за того, что энергия двойной связи $\mathrm{Mg}$ с О больше энергии связи $d$-орбитали материала добавки с $s p^{3}$-орбиталью О, и к тому же $\mathrm{Mg}$ в составе всегда больше, чем добавки. Поэтому естественно, что при увеличении содержания добавки начнется образование в ней $\delta$-связей $\mathrm{Me}-\mathrm{Me}$, характерных для переходных Ме, приводящих к кристаллизации (образованию на этих связях решеток ОЦК, ГЦК или ГПУ типов). Этот процесс исключает часть добавки из состояния субоксида и способствует ее собственному окислению и, как следствие, уменьшает способность материала к поглощению $\mathrm{H}_{2}$. Вот почему для получения максимальной емкости необходимо вполне определенное и к тому же не очень большое количество добавки. В этом можно убедиться на примере составов типа $\mathrm{Mg}_{n} \mathrm{Ni}$ (табл. 1).

Образование субоксида с добавкой переходного Ме, не имеющего вакантных $d$-орбиталей, происходит в основном по описанной схеме, но с небольшим различием. Оно заключается в том, что донорно-акцепторная связь добавки с оксидом $\mathrm{MgO}$ происходит не с его атомом О, a с оставшейся вакантной третьей $s p$-орбиталью Mg. И в этом случае увеличение количества добавки сверх номинального также приводит к уменьшению водородной емкости.

Кроме того, на поверхности не следует исключать и указанное выше явление ее реконструкции при взаимодействии с О. Т.е. нельзя исключать образование на 
поверхности субоксида с $\mathrm{Mg}$ по первому вышеописанному варианту. Тем более уже ясно, что для начала поглощения $\mathrm{H}_{2}$ важно состояние именно поверхности закрытых верхних пор, а затем уже закрытых объемных.

Так как поверхность на фундаментальном уровне определяется как слой атомов вещества в конденсированном состоянии (т.е. жидкости или твердого тела), граничащий, с одной стороны, с атомами объема, а с другой - с вакуумом или средой иной химической природы, стенки объемных дефектов твердого тела (трещин, границ зерен, бороздок, канавок, выходов линейных дислокаций и т.п.) также следует считать поверхностью. Т.е. поверхность вообще на атомном уровне можно считать особым состоянием вещества. Поэтому следует различать поглощение чужеродных атомов в объем кристаллической решетки от поглощения в объем материала. Последнее обычно происходит по дефектам и по большому счету относится все же к явлениям на поверхности.

Деградацию же обратимой емкости для химически подобных Ме добавок, например, Fe и $\mathrm{Ni}$ (для первого она наблюдается, а для второго можно добиться ее отсутствия) при всех прочих равных условиях пока можно объяснить неоптимальным для $\mathrm{Fe}$ количеством добавки, хотя даже весовые соотношения выбирались одинаковыми (типа $\mathrm{Mg}_{10} \mathrm{Me}$ ). Если же экспериментально будет показано, что на ресурсоспособность состава (т.е. на стабильность обратимой емкости) с добавкой без вакантных $d$-орбиталей влияет еще и количество заполненных (у Fe - 6, а у $\mathrm{Ni}-8$ ), то последний фактор станет фундаментальным. Это вполне может составить предмет дальнейших исследований.

Не менее сложной задачей является и установление фундаментального принципа обнаруженного снижения $T_{\text {hyd }}$ при использовании некоторых добавок к $\mathrm{Mg}$. В этой работе подобные исследования не проведены, и они также могут представлять интерес для дальнейших исследований. Ясно, что для прекращения статистического характера исследований, проводимых до сих пор, необходимо разработать фундаментальные принципы получения максимальной обратимой емкости и минимально возможных температур гидрирования рабочего состава твердотельного водородного аккумулятора.

\section{Заключение}

Таким образом, к настоящему времени наиболее подходящим для применения в твердотельных водородных аккумуляторах, в том числе и из экономических соображений, является состав с весовой формулой $\mathrm{Mg}_{10} \mathrm{Ni}$ $(80.6 \% \mathrm{Mg}+19.4 \% \mathrm{Ni})$. На его основе уже вполне можно создавать полномасштабный экспериментальный гидридный бак. В меру высокая оптимальная $T_{\text {hyd }} \mathrm{Mg}_{10} \mathrm{Ni}-$ $650 \mathrm{~K}$ не должна являться препятствием на этом пути, так как на водородозаправочной станции можно заправлять бак предварительно нагретым до необходимой
$T$ водородом. А для почти полного выделения $\mathrm{H}_{2}$ вполне достаточно $T$ выхлопных газов ДВС. При этом различная скорость выделения $\mathrm{H}_{2}$ при различных $T$ выхлопных газов может компенсироваться применением накопительного баллона (ресивера), снабженного обычным автомобильным компрессором, аналогичным применяемому для пневматических тормозов. Напуск же $\mathrm{H}_{2}$ в цилиндры ДВС производится уже из ресивера с помощью газораспределительной системы при необходимом для этого давлении дросселированием. При переменном же (осциллирующем) давлении становится необходимой еще и система автоматического опережения зажигания.

Для дальнейшего усовершенствования водородного аккумулятора необходимо решить указанные выше проблемы деградации обратимой емкости, например, ориентируясь не на весовую, а на брутто-стехиометрическую формулу состава, и, кроме того, исследовать составы при различных плотностях брикетов для выбора оптимальной начальной плотности с учетом „распухания“ при гидрировании для того, чтобы получить минимальный объем гидридного бака. Некоторые исследования в этой области нами уже проведены (табл. 3).

Приведем данные о требуемом количестве аккумулирующего $\mathrm{H}_{2}$ состава для пробега автомобиля в $300 \mathrm{~km}$. Для этого требуется $5 \mathrm{~kg} \mathrm{H} 2[11,15]$. С учетом плотности $\mathrm{H}_{2}$ в $0.08987 \mathrm{~g} / \mathrm{dm}^{3}$ получаем, что при обратимой емкости состава $3001 / \mathrm{kg}$ (2.7 mass. \%) требуется $\sim 186 \mathrm{~kg}$ состава. Соответственно для $4001 / \mathrm{kg}$ (3.59 mass. \%) $\sim 139 \mathrm{~kg}$, для $5001 / \mathrm{kg} \quad(4.49$ mass. $\%) \sim 111 \mathrm{~kg}$, для $6001 / \mathrm{kg}(6.39$ mass. $\%) \sim 93 \mathrm{~kg}$, для $7001 / \mathrm{kg}$ (6.29 mass. \%) $\sim 80 \mathrm{~kg}$ и для $7501 / \mathrm{kg}(6.74$ mass. $\%) \sim 75 \mathrm{~kg}$.

\section{Выводы}

Исходя из результатов этой работы можно прийти к следующим выводам и рекомендациям:

- компактный $\mathrm{Mg}$ и сильно спрессованный его порошок $\mathrm{H}_{2}$ не поглощают;

- следует определить оптимальную дисперсность порошка. Он не может быть чрезмерно мелким во избежание ухудшения теплопроводности и, как следствие, выхода из оптимального режима гидрирования;

- основным фактором поглощения $\mathrm{H}_{2}$ является окклюзия, а не образование гидрида;

- прессование порошка в брикет до определенной степени не влияет на водородную емкость и с учетом „распухания“ при гидрировании необходимо лишь для оптимальной загрузки гидридного бака;

- широко исследовавшийся ранее состав $\mathrm{Mg}_{2} \mathrm{Ni}$ не является оптимальным по водородной емкости; - добавка к $\mathrm{Mg}$ необходима для сохранения поверхностью электропроводности и снижения Т гидрирования; - наиболее желательна добавка к $\mathrm{Mg}$ из переходного $\mathrm{Me}$, имеющего вакантные $d$ - или $f$-орбитали атомов ( $\mathrm{Sc}$, Y, La,Ti, Zr, Hf, V, Nb, Ta, Ce, Pr, Nd, Pm, Sm); 
- из экономических соображений может быть выбрана добавка к $\mathrm{Mg}$ из переходных Ме, имеющих заполненными и (или) полузаполненными все $d$-орбитали атомов (Cr, Mo, W, Mn, Fe, Co, Ni);

- соотношение $\mathrm{Mg}$ и добавки для получения оптимального состава должно быть вполне определенным;

- от весовой формулы для выбора состава следует отказаться в пользу стехиометрической;

- составу с максимальной обратимой водородной емкостью без ее деградации соответствует минимальная $T$ гидрирования.

\section{Конфликт интересов}

Авторы заявляют, что у них нет конфликта интересов.

\section{Список литературы}

[1] Подгорный А.Н., Варшавский И.Л., Макаров А.А., Мищенко А.И. Применение водородного топлива в двигателях внутреннего сгорания. Препринт-45. ИП Маш АН УССР. Харьков, 1977. 32 с.

[2] Варшавский И.Л., Мищенко А.И., Степанов В.Ю. Малотоксичный Автомобиль. Сб. докл. Всезоюзн. конф. „Защита воздушного бассейна от загрязнения токсичными выбросами транспортных средств“. Харьков: Изд-во ИП Маш АН УССР, Т. 1. Харьков 1977. С. 83-92.

[3] Варшавский И.Л. Применение водорода в тепловых двигателях. Атомно-водородная энергетика и технология. Вып. 3. М.: Атомиздат, 1980. 272 с.

[4] Кузнецов Н.Д. Использование водорода в качестве топлива в авиации. Атомно-водородная энергетика и технология. Вып. 3. М.: Атомиздат, 1980. 272 с.

[5] Мищенко А.И. Применение водорода для автомобильных двигателей. Киев: Наукова думка, 1984. 143 с.

[6] Коротеев А.С., Миронов В.В., Смоляров В.А. // Альтернативная энергетика и экология. 2004. № 1. С. 5-13.

[7] Вахрушев А.В., Яновский Ю.Г., Шестаков И.А., Федотов А.Ю. Альтернативная энергетика и экология. 2004. 34. C. 41-48.

[8] Байбиков А.С. // Альтернативная энергетика и экология. 2009. № 10. C. $30-32$.

[9] Топливо из воды. Московская правда. № 247(16954) от 21.10.75.

[10] Конарев Ф.М. Вода - новый источник энергии. 3-е изд. Краснодар, 2001. 200 с.

[11] Водород. Свойства, получение, хранение, транспортирование, применение (справочник). / Под ред. Д.Ю. Гамбурга, Н.Ф. Дубовкина. М.: Химия, 1989. 672 с.

[12] Мищенко А.И., Черкашин Ю.В. // Автомобильная промышленность, 1980. № 9. С. 21-28.

[13] Черников А.С., Фадеев В.Н., Савин В.И. Атомноводородная энергетика и технология. М.: Атомиздат, 1980. Вып. 3. 272 c.

[14] Шанин Ю.И. // Альтернативная энергетика и экология. 2002. № 3. C. $50-53$.

[15] Шиильрайн Э.Э., Малышенко С.П., Кулешов Г.Г. Введение в водородную энергетику. М.: Энергоатомиздат, 1984. 264 c.
[16] Carter G.C., Carter F.L. // Metal-hydrogen systems. / Ed. T.N. Veziroglu. Pergamon Press, 1982. P. 503-529.

[17] Turillon P.P. Hydr. Energ. Progress IV. / Ed. T.N. Veziroglu. Pergamon Press, 1982. Vol. 3. P. 1289-1305.

[18] Buchner H. Hydr. Energ. Progress IV. / Ed. T.N. Veziroglu, W. Seifritz. Pergamon Press, 1978. Vol. 4. P. 1749-1792.

[19] Антонова М.M. Соединения магния - аккумуляторы водорода. Препринт 93-№ 1. АН Украины. Ин-т проблем материаловед. Киев, 1992. 41 с.

[20] Захаров А.И., Иванков Вас.В., Иванков Вяч.В., Терехов В.Н., Милованова О.A. // ISJAEE. 2002. № 2. С. 20-24.

[21] Тарасов Б.П., Фокин В.Н., Борисов Д.Н., Гусаченко Е.И., Клямкин С.Н., Яковлева Н.А., Шилкин С.П. // ISJAEE. 2004. № 1 (9). C. 47-52.

[22] Тарасов Б.П. // ISJAEE. 2006. № 2(34). С. 11-17.

[23] Ажсажа В.М., Тихоновский М.А., Шепелев А.Г., Курило Ю.П., Пономаренко Т.А., Виноградов Д.В. // ВАНТ. 2006. № 1. C. $145-152$.

[24] Металлогидридные аккумуляторы водорода многократного // действия. ISJAEE. 2008. № 3 (59). С. 160.

[25] Belkbir L., Gerard N., Percheron-Guegan A. // Intern. J. Hydr. Energ. 1978. Vol. 4. P. 541-557.

[26] Джсамагидзе Ш.З., Зыков Б.М., Сабо Е.П., Швангирадзе P.P. // Наука и технологии в промышленности. 2008. № 4. C. 35-37.

[27] Дымова Т.Н., Стерлядкина З.К., Сафронов В.Г. // ЖНХ 1961. Т. 6. Вып. 4. С. 763-767.

[28] Гидриды металлов. / Под ред. В. Мюллера, Д. Блекледжа, Дж. Либовица. М.: Атомиздат, 1973. 426 с.

[29] Гидриды переходных металлов. / Под ред. Э.М. Мьюттериза. М.: Мир, 1975. $311 \mathrm{c.}$

[30] Антонова М.М., Морозова Р.А. Препаративная химия гидридов. Справочник. Киев. Наукова думка, 1976. 98 с.

[31] Водород в металлах. / Под ред. Г. Алефельда, И. Фелькля. Т. 2. Прикладные спектры. М.: Мир, 1981. Т. 1. 480 с; Т. 2. $432 \mathrm{c}$.

[32] Бульчев Б.М., Сторожсенко П.А. // ISJAEE. 2004. № 4. C. $5-10$.

[33] Физико-химические свойства элементов. / Под ред. Г.В. Самсонова. Киев, Наукова думка, 1965. 808 с.

[34] Зыков Б.М., Иконников Д.С., Цхакая В.К. // ФТТ. 1975. т. 17. Вып. 1. С. 274-279.

[35] Зыков Б.М., Иконников Д.С., Цхакая В.К. // ФТТ. 1977. Т. 19. Вып. 12. С. 3672-3676.

[36] Зыков Б.М., Кобяков В.П., Нардая Ю.И. // Высокочистые вещества. 1991. № 1. С. 71-80.

[37] Корнилов И.И., Глазова В.В. Взаимодействие тугоплавких металлов переходных групп с кислородом. М.: Наука, 1967. $256 \mathrm{c}$.

[38] Зыков Б.М., Сабельников А.М. // Поверхность. 1988. № 10. C. $61-70$.

[39] Carrol J.J., Melmed A.J. // Surf. Sci. 1969. Vol. 16. P. $251-264$.

[40] Бравареи, А.В., Зыков Б.М., Зыкова В.Н., Лебедев В.Н., Удовиченко Ю.К. // ЖТФ. 2002. Т. 72. Вып. 9. С. 112-118.

[41] Зыков Б.М., Зыкова В.Н., Лебедев В.Н., Удовиченко Ю.К. // ЖТФ. 2002. Т. 72. Вып. 6. С. 90-100.

[42] Кукушкин Ю.Н., Маслов Е.И. Строение атома и химическая связь. Л.: Изд-во Лен. ун-та, 1973. 80 с. 